\title{
Which dimensions of disability severity does the HIV Disability Questionnaire (HDQ) measure? An exploratory factor analysis
}

\author{
Kelly K. O’Brien, Ahmed M. Bayoumi, Paul Stratford \\ and Patricia Solomon
}

Version Post-Print/Accepted Manuscript

\begin{abstract}
Citation O`Brien KK, Bayoumi AM, Stratford P, Solomon P. Which dimensions (published version) of disability severity does the HIV Disability Questionnaire (HDQ) measure? An exploratory factor analysis. Disability and Rehabilitation. 2015;37(13):1193-201. doi: 10.3109/09638288.2014.949358. Epub 2014 Aug 13.
\end{abstract}

Publisher's Statement The Version of Record of this manuscript has been published and is available in Disability and Rehabilitation 2014.

http://www.tandfonline.com/10.3109/09638288.2014.949358.

How to cite TSpace items

Always cite the published version, so the author(s) will receive recognition through services that track citation counts, e.g. Scopus. If you need to cite the page number of the TSpace version (original manuscript or accepted manuscript) because you cannot access the published version, then cite the TSpace version in addition to the published version using the permanent URI (handle) found on the record page. 
Which dimensions of disability does the HIV Disability Questionnaire (HDQ) measure?

\section{A factor analysis}

Kelly K. O'Brien ${ }^{1,2}$

Ahmed M. Bayoumi ${ }^{3,4,5}$

Paul Stratford ${ }^{2}$

Patricia Solomon $^{2}$

${ }^{1}$ Department of Physical Therapy, University of Toronto 500 University Avenue, Room 160

Toronto, Ontario, M5G 1V7

Canada

${ }^{2}$ School of Rehabilitation Science, McMaster University 1400 Main Street West, Room 408

Hamilton, Ontario, L8S 1 C7

Canada

${ }^{3}$ Centre for Research on Inner City Health,

Keenan Research Centre of the Li Ka Shing Knowledge Institute, St. Michael's Hospital, 30 Bond Street, Toronto, Ontario, M5B 1W8

Canada

${ }^{4}$ Institute of Health Policy, Management and Evaluation, University of Toronto, Toronto, Ontario, Canada

${ }^{5}$ Department of Medicine, University of Toronto, Toronto, Ontario, Canada

\section{Corresponding Author}

Kelly O'Brien

Department of Physical Therapy, University of Toronto

160-500 University Avenue

Toronto, Ontario M5G 1V7

Canada

Phone: 1-416-978-0565

Fax: 1-416-946-8562

kelly.obrien@utoronto.ca 


\title{
Which dimensions of disability does the HIV Disability Questionnaire (HDQ) measure? A factor analysis
}

\begin{abstract}
Purpose: To assess the dimensions of disability measured by the HIV Disability Questionnaire (HDQ), a newly developed 72 item self-administered questionnaire that describes the presence, severity and episodic nature of disability experienced by people living with HIV.
\end{abstract}

Methods: We recruited adults living with HIV from hospital clinics, AIDS service organizations and a specialty hospital and administered the HDQ followed by a demographic questionnaire.

We conducted an exploratory factor analysis using disability severity scores to determine the domains of disability in the HDQ. We used the following steps: a) ensured correlations between items were $>0.30$ and $<0.80$; b) conducted a principal components analysis to extract factors; c) used the Scree Test and eigenvalue threshold $>1.5$ to determine the number of factors to retain; and d) used oblique rotation to simplify the factor loading matrix. We assigned items to factors based on factor loadings of $>0.30$.

Results: Of 361 participants, $80 \%$ were men and 77\% reported living with at least two concurrent health conditions in addition to HIV. The exploratory factor analysis suggested retaining six factors. Items related to symptoms and impairments loaded on three factors (physical [20 items], cognitive [3 items], and mental and emotional health [11 items]) and items related to worrying about the future, daily activities, and personal relationships loaded on three additional factors (uncertainty [14 items], difficulties with day-to-day activities [9 items], social inclusion [12 items]). 
1

2

3

4

5

6

7

8

9

10

11

12

13

14

15

16

17

18

19

20

21

22

23

24

25

26

27

28

29

30

31

32

33

34

35

36

37

38

39

40

41

42

43

44

45

46

47

48

49

50

51

52

53

54

55

56

57

58

59

60
Conclusions: The HDQ has six domains: physical symptoms and impairments; cognitive symptoms and impairments; mental and emotional health symptoms and impairments; uncertainty; difficulties with day-to-day activities and challenges to social inclusion. These domains establish the scoring structure for the dimensions of disability measured by the HDQ.

URL: http:/mc.manuscriptcentral.com/dandr Email: davemuller@suffolk.ac.uk 


\section{INTRODUCTION}

As individuals live longer and age with HIV, many are living with the health-related consequences of HIV and its comorbidities [1-6], a concept collectively referred to as disability. In an earlier qualitative study, a conceptual framework of disability was derived from the perspective of 38 adults living with HIV. Participants broadly defined disability as any physical, cognitive, mental or emotional symptoms and impairments, difficulties with day-to-day activities, challenges to social inclusion, and uncertainty or worrying about the future that an individual may experience living with HIV [7]. Validated in an former phase of research, the Episodic Disability Framework consists of three main components: 1) dimensions of disability (symptoms and impairments; difficulties carrying out day-to-day activities; challenges to social inclusion; and uncertainty about future health), that may fluctuate on a daily basis and over the entire course of living with HIV, 2) contextual factors (social support, stigma, living strategies and personal attributes) that may exacerbate or alleviate the dimensions of disability, and 3) triggers, defined as life events that mark momentous or major episodes of disability $[7,8]$. This Framework provided a new way to conceptualize disability from the perspective of adults with HIV.

Measuring disability is critical for determining the impact of the disease and associated comorbidities and for evaluating the effectiveness of interventions that might reduce disability. While health status questionnaires are widely used in HIV research [9-13], a previous content analysis of HIV-specific instruments suggested that instruments developed prior to the advent of combination antiretroviral therapy do not capture the breadth of disability experienced by adults living with HIV, particularly domains related to social inclusion and uncertainty [14]. Although more recently developed quality of life instruments capture dimensions of social relationships, 
work, and future $[15,16]$, they were not developed for the purpose of assessing the construct of disability. Hence, there was a need for a new instrument to comprehensively describe disability experienced by adults living with HIV.

We developed a new HIV-specific disability questionnaire using the Episodic Disability Framework [17]. The HIV Disability Questionnaire (HDQ) is a 72-item self-administered questionnaire developed for the purpose to describe the presence, severity and episodic nature of disability experienced by adults living with HIV. Development of a new questionnaire requires multiple stages of comprehensive and rigorous assessment and refinement. We previously demonstrated that the HDQ has face and content validity and is easy to use with adults living with HIV [18]. However, the true domains of disability (or scoring structure) of this instrument was unknown. Knowing the domain and scoring structure is critical to the development of an instrument to ensure that clinicians and researchers are able to adequately score the questionnaire and interpret domain scores to accurately assess the dimensions of disability experienced by adult living with HIV. Our aim was to assess the dimensions of disability (or domain structure) of the HDQ among adults living with HIV.

\section{METHODS}

We recruited adults (18 years and older) living with HIV from hospital clinics, AIDS service organizations and a specialty hospital. We administered the HDQ, followed by a demographic questionnaire. All aspects of this project were conducted in collaboration with a Community Advisory Committee. This research was approved by Research Ethics Boards at the University of Toronto, and McMaster University. 


\title{
HIV Disability Questionnaire (HDQ)
}

The HDQ consists of items that describe the presence, severity and episodic nature of disability experienced by adults living with HIV. Items in the HDQ were generated from detailed categories of the four disability dimensions in the Episodic Disability Framework [7] and supplemented by our previous content analysis of existing health status instruments [14] and by input from members of the Community Advisory Committee. In an earlier assessment of HDQ sensibility, adults living with HIV and clinicians who work in HIV care supported the term 'disability' representing the construct of interest in the HDQ. Disability collectively represented the health-challenges defined by adults living with HIV described in the Episodic Disability Framework [18].

A typical HDQ item presents a statement about a health-related challenge (for example, "I feel too tired to do my usual activities") and has both a seven point ordinal response scale asking the respondent to rate the challenge on the day of administration (from 0 -'Not at all' to 6 -'Extremely') and a nominal response scale asking whether the challenge fluctuated over the past week ('Yes' or 'No'). The focus of our analysis was on the HDQ severity scale.

\begin{abstract}
Analysis
We conducted an exploratory factor analysis using disability severity scores to determine the underlying factor (domain) structure of the HDQ and to determine any items that may be redundant and considered for deletion. An exploratory approach is commonly used in early stages of questionnaire development for assessing domain structure and item reduction [19].

We used a simple summative approach to calculate the severity scores of the HDQ.
\end{abstract} Severity scores were calculated by summing individual item scores (ranging from 0 to 6 ) for 
each domain and then we linearly transformed the domain scores out of a scale from 0 to 100 . Higher scores indicated a greater severity of disability.

Our factor analysis included the following steps [20]. First, we assessed whether the correlation matrix of HDQ items was factorable using a combination of four methods: a) ensuring correlations between items were $>0.30$ and $<0.80$; b) ensuring communalities were $>0.60$. Communalities are a feature of questionnaire items that represent the proportion of variance in each individual item shared across all the factors [21]; c) ensuring the Kaiser-Meyer Olkin (KMO) measure of sampling adequacy was $>0.90$, which would suggest that the items explain common variance with other items, suggesting underlying common factors [22]; and d) assessing whether individual measures of sampling adequacy were $>0.70$, which would indicate that the correlations among individual items are strong enough to suggest the matrix is factorable [19].

Second, we conducted a principal components analysis to guide data reduction [23]. We extracted factors (or components) using an iterative process whereby the first factor accounted for the highest proportion of variance among item scores of the HDQ, the second extracted factor accounted for the second highest proportion of remaining variance, and so forth, until the last factor accounted for the least proportion of variance.

Third, we determined the number of factors to retain using a combination of eigenvalues and the Cattell Scree Plot. Eigenvalues are numbers that represent the amount of variance in all items explained by a given factor. Although a threshold eigenvalue of 1.0 has commonly been used, this approach tends to result in retaining a greater number factors than needed with instruments with more than 50 items [24]. Accordingly, we used a threshold eigenvalue of 1.5. Scree plots are recommended to help determine the factor structure and number of factors to 
retain in a solution [25]. The Cattell Scree Test involves plotting eigenvalues against each factor. The scree portion of the plot is the point where the slope changes from negative to almost zero indicated by a sharp break in the curve. Factors are retained up to the point prior to the scree [26].

Fourth, we assessed the factor loading matrix, which consists of the correlation of each item with each of the retained factors. When the factor loading matrix was not simple (meaning each HDQ item loaded on more than one factor and each factor possessed high loadings for multiple items), we conducted an oblique rotation of the factors to produce a structurally simpler matrix to assist with interpretation $[19,25]$. We chose oblique rotation because dimensions of disability in the HDQ are considered conceptually related (or correlated) to collectively comprise the construct of disability.

We interpreted our results by assessing the factor pattern matrix to determine the extent to which items in the HDQ loaded on a given factor. Floyd and Wideman (1995) suggest that factor loadings are generally considered meaningful when they exceed either 0.3 or 0.4 . We defined factor loadings $>0.30$ as indicating a relationship between an item on the HDQ and a given factor. These items were considered as 'loading' on that factor to ensure we did not remove any items prematurely that contribute to the measurement of disability [23]. Items that loaded on more than one factor even after oblique rotation were allocated to a factor (or disability domain) based on our clinical judgment and conceptual understanding [19]. Items with no factor loadings above 0.30 were considered as contributing little to the overall questionnaire and, if clinically sensible, were considered for deletion. Items loading on the same factor with loadings $>0.80$ may suggest conceptual similarity across the items and were considered for removal to minimize redundancy of items [19]. We used the Episodic Disability Framework and 
consultations with our Community Advisory Committee to inform the final allocation of items to factors and naming of the factors (or domains) of the HDQ.

Our target sample size was calculated using the rule of thumb that a minimum of five participants per item are required for factor analysis [27]. With 72 disability severity items in the HDQ, we required at least 360 participants. We used SPSS (version 19.0) for data manipulation and SAS (version 9.2) for all analyses [28, 29].

\section{RESULTS}

Three-hundred and sixty-one participants were recruited from AIDS service organizations in Toronto (51\%); word of mouth (28\%), AIDS service organizations in Hamilton, Niagara and Durham regions (7\%), a specialty hospital (3\%) and hospital clinics in Toronto and Hamilton, Ontario (3\%). The majority of participants were men (80\%), the median age was 46 years, most were taking antiretroviral medications $(83 \%)$, and many $(77 \%)$ were living with two or more health conditions in addition to HIV (Table 1).

\section{Insert table 1 about here}

\section{$H D Q$}

The median time to complete the HDQ was 14 minutes $\left(1^{\text {st }}-3^{\text {rd }}\right.$ quartile: $10-20$ minutes $)$. When characterizing the severity of items, few participants selected response options ' 2 ' and ' 4 ', which did not have a word description. Almost all HDQ items $(n=67)$ demonstrated a floor effect with $>15 \%$ of responses rated as ' 0 ' indicating no disability. Floor effects were most common in items that referred to symptoms and impairments or difficulties with day-to-day activities. Ten HDQ items demonstrated a ceiling effect with $>15 \%$ of responses rated ' 6 ' indicating the highest 
severity of disability. Ceiling effects were most common in items pertaining to worrying about the future. The median HDQ severity score (range $0-100)$ was $31\left(1^{\text {st }}-3^{\text {rd }}\right.$ quartile: $\left.19-45\right)$.

\section{Factor Analysis Results}

Overall results from the factor analysis suggest the HDQ possesses six domains: mental and emotional health symptoms and impairments (Factor 1); uncertainty (Factor 2); difficulties with day-to-day activities (Factor 3); physical symptoms and impairments (Factor 4), cognitive symptoms and impairments (Factor 5), and challenges to social inclusion or interactions with others (Factor 6). We provide a detailed overview of results from each stage of the analysis that led to these findings.

All HDQ items correlated with at least one other item $(0.30<\mathrm{r}<0.80)$ except for item \#18 (I am gaining weight) (correlation matrix available upon request). All communalities were $>0.60$. The KMO measure of sampling adequacy was 0.94. All HDQ items possessed individual measures of sampling adequacy $>0.70$ except for item $\# 18$, which had a sampling adequacy of 0.52. Given these results, we removed item \#18 from the remainder of the factor analysis.

The eigenvalue scores indicated retaining six factors and the Cattell Scree Plot (not shown) indicated retaining either two or six factors. Hence, we retained six factors in the final solution. After oblique rotation, the factor loading matrix yielded a simpler structure (Table 2). At least three items loaded on each retained factor [30]. Two items did not load $>0.30$ on any factors (item \#3: I have constipation; and item \#66: I struggle to maintain safe and stable housing living with HIV). Item \#29 (I am afraid for my future) loaded on two factors with loadings $>0.40$ (Table 2). Twelve other items loaded on two factors with loadings $>0.30$. Fourteen items had factor loadings on more than one factor with less than $<0.20$ absolute difference between 
loadings. Three items (items \#40, 41 and 42) had factor loadings $>0.80(0.81,0.85$ and 0.84 respectively) suggesting potential redundancy [19].

\title{
Insert table 2 about here
}

\begin{abstract}
We conducted post hoc analyses with two, four, five and seven factor structures (not shown) to confirm the number of factors to retain. The six domain structure was the solution that had the least number of items that did not load on any factors and the least number of items that crossloaded on multiple factors.
\end{abstract}

\section{Factor Interpretation}

The majority of items that loaded on Factor1 appeared to refer to mental and emotional health symptoms and impairments (items \#27-37; e.g. feeling anxious, feeling sad, down or depressed, feeling guilty). Other items that loaded on this factor appeared to refer to challenges to social inclusion such as personal relationships, work and school. These items tended to start with 'I feel' (item \#63, 65) or 'I find it hard to...' (items \#62, 68, 69, 70, 72). Item \#29 (I am afraid for my future) loaded on both Factors 1 and $2>0.40$ (Table 2).

The majority of items that loaded on Factor 2 appeared to refer to uncertainty and worrying about the future living with HIV (items \#38-48). However, three items that referred to worrying about the future specifically in reference to 'others' loaded on Factor 6 (items \#49-51). Item \#52 (I have put certain life decisions on hold because of my uncertainty living with HIV) loaded on both Factors 1 and 2 ( $>0.30)$ (Table 2$)$.

The majority of items that loaded on Factor 3 appeared to refer to difficulties carrying out day-to-day activities (items \#53-58; item \#60). Items \#62, \#64 and \#65 that referred to aspects of social inclusion such as volunteering, work or school loaded on Factor 3 as well as Factor 1 with 
the other social inclusion items that started with 'I find it hard to...' or 'I feel'. Item \#12 (I am unsteady on my feet) also loaded on Factor 3 (0.67). Items that referred to work, school or volunteering (items \#62, 64, 65) cross-loaded on Factors 1 and 3 (>0.30) (Table 2).

The majority of items that loaded on Factor 4 appeared to refer to physical symptoms and impairments (items \#1, 2, 4-9, 11, 13-16, 20, and 24-26) (Table 2).

The majority of items that loaded on Factor 5 appeared to refer to cognitive symptoms and impairments (item \#21-23) including difficulty remembering, thinking clearly or concentrating. Item \#17 (I am losing weight), item \#19 (I lack an appetite for food), and item \#59 (I have trouble keeping track of my finances) also loaded on this Factor $(>0.30)$ (Table 2).

Items that loaded on Factor 6 appeared to include items that referred to challenges to social inclusion as it related to interactions with 'others'. For example, items \#49-51 referred to worrying about legal issues of disclosing HIV status to others, worrying about what others will think, and worrying about transmitting HIV to others. Item \#67 also referred to difficulty talking with 'others' (Table 2).

All factors were correlated with each other at $\geq 0.30$ with the exception of Factor 6 which correlated with Factor $3(\mathrm{r}=0.17)$, Factor $4(\mathrm{r}=0.16)$, and Factor $5(\mathrm{r}=0.24)$. Table 3 indicates the final domain structure of the HDQ: physical symptoms and impairments (20 items); cognitive symptoms and impairments (3 items); mental and emotional health symptoms and impairments (11 items); uncertainty (14 items); difficulties with day-to-day activities (9 items); and challenges to social inclusion (12 items) (Table 3).

Insert table 3 about here 


\section{DISCUSSION}

The HDQ appeared to have six domains: mental and emotional health symptoms and impairments (Factor 1); uncertainty (Factor 2); difficulties with day-to-day activities (Factor 3); physical symptoms and impairments (Factor 4), cognitive symptoms and impairments (Factor 5), and challenges to social inclusion or interactions with others (Factor 6).

Because the factor analysis did not yield a simple structure, we used the Episodic Disability Framework and consulted with our Community Advisory Committee to inform considerations for HDQ revision and the recommended domain structure of the HDQ (Table 3).

Factor analysis results suggested the HDQ possessed three types of symptoms and impairments. This domain structure is supported by evidence that suggests that physical, mental and emotional health are related but distinct concepts requiring unique approaches to treatment for people living with HIV [31]. Item \#17 (I am losing weight), item \#18 (I have trouble sleeping), item \#42 (I have problems with my vision), and item \#59 (I have trouble keeping track of my finances) also loaded on the cognitive symptoms and impairments domain (Factor 5) which suggests their relation to cognitive health. Nevertheless, we decided that these items more closely represented physical and social health challenges with HIV.

Items that referred to challenges to social inclusion often loaded across multiple factors (Factors 1, 3 and 6). Items that pertained to social inclusion, but started with the terms 'I feel' or 'I find it hard to...' tended to load on Factor 1 (mental and emotional symptoms and impairments domain), whereas items that included the term 'others' tended to load on Factor 6, representing challenges to social inclusion. Cross-loading of social inclusion items on multiple factors may be explained by the influence of physical symptoms and impairments and challenges with day-to- 
day activities on social inclusion. Additionally, we might expect items with similar wording that refer to emotions to load together even though they exist within the context of social inclusion.

Challenges with factor interpretation may be attributed to our use of a psychometric approach of factor analysis to establish the HDQ domain structure, which differed from our prior clinimetric approach to HDQ development and sensibility assessment. We generated HDQ items using the Episodic Disability Framework, and assessed sensibility from the perspective of people with HIV and clinicians who work in HIV care $[17,18]$. Nevertheless, we feel using a combined approach will yield the most accurate and relevant domain structure of the HDQ [32, 33]. Future confirmatory factor analysis will establish overall goodness of fit of the items with the new domain structure.

Although the housing item did not load on any factor, this question was generated by the Community Advisory Committee who felt we should retain this item given the importance of housing in relation to the physical and mental health of people living with HIV [34].

The lack of endorsement for response options ' 2 ' and '4' on the HDQ severity scale was most likely due to the lack of associated word descriptions. [35] Accordingly, it seems prudent to reduce the number of HDQ response options from 7 to 5; this might diminish reliability but would still meet the recommended number of options for health measurement scales. [35]

Floor and ceiling effects were apparent across the HDQ. The ceiling effect, primarily seen in items that addressed day-to-day activities, was likely a reflection of our sample, adults living with HIV independently in the community with few challenges in mobility and self-care activities. Severe skewness of item scores may deflate standard correlation coefficients [36] which could have led to an underestimation of factor loadings in our analysis. 
Our analysis led to few situations of item deletion. Many of the items loaded weakly on the primary factor, and fourteen items loaded on multiple factors without more than a 0.20 difference between loadings, which is the rule of thumb for an item to be considered theoretically meaningful on the factor with the highest loading [37]. Nevertheless, we chose to retain items at this stage of the analysis given their theoretical relevance to disability. Items that referred to worrying about having an illness (item \#40), when the illness might occur (item \#41) and what the outcome of the illness might be (item \#42) all possessed factor loadings greater than 0.80 suggesting potential redundancy and opportunities for item reduction. We suggest removing item \#41 because it possessed the highest factor loading (0.85) and the Community Advisory Committee felt worrying about having an illness and the outcome of that illness contributed more to uncertainty than worrying about when the illness might occur. Overall, we approached decisions of item deletion as a balance between the psychometric and clinimetric approaches, siding with item retention in consultation with the Community Advisory Committee.

In the final domain structure of the HDQ, we allocated item \#12 (I am unsteady on my feet) to the difficulties with day-to-day activities domain (Table 3). This item loaded strongly on this factor (0.67) and only weakly on the physical symptoms and impairments factor $(0.21)$ and closely relates to an individual's mobility, which is a large component of the difficulties with day-to-day domain. No other re-allocation of items solely based on factor loadings made conceptual or clinical sense.

Item \#29 (I am afraid for my future) was the only item that cross loaded on two factors, Factor 1 (mental and emotional symptoms and impairments) and Factor 2 (uncertainty) $>0.40$. This item addresses fear (an emotion) and places it in relation to worrying about the future (uncertainty). All other situations of cross loadings were $>0.30$ and the majority of factor 
loadings across factors did not greatly differ. Hence, we allocated items as conceptualized in the Episodic Disability Framework and Community Advisory Committee.

Dimensions of disability in the HDQ may overlap with other health-related concepts such as symptoms and health-related quality of life (HRQL) measured in the context of HIV. Recent HIV-related quality of life questionnaires include items related to social relationships, work, and future that may be analogous to the HDQ domains of challenges to social inclusion and uncertainty respectively $[15,16]$. However, HRQL instruments were not developed to assess the episodic nature of disability. Furthermore, components of HRQL such as personal goals, values and expectations go beyond the scope of disability. Evidence of weak relationships between symptoms and function (domains of disability) and overall health-related quality of life suggest HRQL and disability while related, are distinct concepts, and equally important to consider in the context of HIV $[38,39]$. Adults living with HIV and clinicians who work in HIV supported the term 'disability' to capture the construct of interest in the HDQ because it collectively represented the health-challenges as defined by adults living with HIV in the earlier developed conceptual framework.

\section{Implications for Future Practice}

The HDQ was developed to assess the spectrum of disability experienced by adults living with HIV. Upon further measurement property assessment, researchers may use the HDQ to document the presence, severity and episodic nature of disability experienced by adults living with HIV. Once shown to be responsive and reliable, the HDQ may be considered for use by clinicians to assess the profile of disability experienced by clients, particularly those dimensions that may not have been previously well captured in existing health status instruments such as living with uncertainty. This may help to identify areas in which clinicians and community-based 
organizations might implement programs, services and intervention to reduce disability experienced by their clients with HIV.

Our study has limitations. First, the majority of the sample included men living with HIV recruited from AIDS Service Organizations $\backslash$ in a large metropolitan city. The properties of the HDQ among adults who do not access such organizations or who live in rural areas or the developing country context are unknown. Second, our goal was to assess the domain structure of the HDQ (not measure disability) so HDQ scores should be interpreted cautiously given the meaning of the HDQ scores are unknown. Third, the Episodic Disability Framework provided a preliminary conceptual foundation for the composition of disability. While confirmatory factor analysis or item response theory may have been alternative approaches, we chose an exploratory factor analysis because it is preferred in early stages of questionnaire development [37]. This approach enabled us to further refine our understanding of disability as comprised by six domains [19]. Lastly, no universal standardized method exists to direct the factor analytical process such as factor rotation, determining the number of factors to retain, factor interpretation, and item reduction [40]. While we established an a priori process for guiding our decisionmaking process, the resulting factor loading matrix was not simple. The Episodic Disability Framework and consultations with the Community Advisory Committee were integral to establishing our decisions surrounding item allocation, factor interpretation and item reduction. Also, twelve of the fifteen inter-factor correlations were correlated with each other $\geq 0.30$ which supported our use of oblique rotation. In addition, post hoc analyses confirmed the six domain structure was the optimal solution with the fewest number of items that did not load on any factors, the fewest number of items that cross-loaded, at least three items that loaded on each factor, and made the most clinical sense. 


\section{CONCLUSIONS}

The HDQ is an HIV-specific instrument developed to describe the presence, severity and episodic nature of disability. Results suggest the HDQ has six domains: physical symptoms and impairments (20 items); cognitive symptoms and impairments (3 items); mental and emotional health symptoms and impairments (11 items); uncertainty (14 items); difficulties with day-to-day activities (9 items); and challenges to social inclusion (12 items). These domains establish the scoring structure for the dimensions of disability measured by the HDQ.

\section{ACKNOWLEDGEMENTS}

We acknowledge Marilyn Swinton for her contributions with recruitment and data collection as Research Coordinator on this phase of the HIV Disability Questionnaire Study.

We gratefully acknowledge the members of the Community Advisory Committee including Ken King (Canadian Working Group on HIV and Rehabilitation), James Murray (Ontario Ministry of Health and Long-Term Care), Rob Alexander (HIVER Consulting) and Shane Patey (Toronto People with AIDS Foundation) for their contributions to this work.

We gratefully acknowledge Community Collaborators in this work including Casey House, Toronto People with AIDS Foundation, Canadian Working Group on HIV and Rehabilitation, McMaster Special Immunology Services (SIS) Clinic, the AIDS Network (Hamilton), AIDS Niagara, AIDS Committee of Durham Region, and AIDS Committee of Toronto. 


\section{DECLARATION OF INTEREST}

This research was supported by an operating grant from the Canadian Institutes of Health Research (CIHR) HIV/AIDS Community Based Research Program (CBR\#104072). Dr. Kelly O'Brien was supported by a Fellowship from the CIHR, HIV/AIDS Research Program and Michael G. DeGroote Postdoctoral Fellowship (McMaster University). Dr. Ahmed Bayoumi was supported by a Canadian Institutes of Health Research / Ontario Ministry of Health and Long-Term Care Applied Chair in Health Services and Policy Research. The Centre for Research on Inner City Health is supported in part by a grant from the Ontario Ministry of Health and Long-Term Care. The views expressed in this article are those of the authors, and no official endorsement by supporting agencies is intended or should be inferred. 


\section{REFERENCES}

1. Rusch M, Nixon S, Schilder A, Braitstein P, Chan K, Hogg RS. Impairments, activity limitations and participation restrictions: prevalence and associations among persons living with HIV/AIDS in British Columbia. Health Qual Life Outcomes 2004;2:46.

2. Gaidhane AM, Zahiruddin QS, Waghmare L, Zodpey S, Goyal RC, Johrapurkar SR. Assessing self-care component of activities and participation domain of the international classification of functioning, disability and health (ICF) among people living with HIV/AIDS. AIDS Care 2008;20:1098-1104.

3. Weiss JJ, Osorio G, Ryan E, Marcus SM, Fishbein DA. Prevalence and patient awareness of medical comorbidities in an urban AIDS clinic. AIDS Patient Care STDS 2010;24:3948.

4. Willard S, Holzemer WL, Wantland DJ, et al. Does "asymptomatic" mean without symptoms for those living with HIV infection? AIDS Care 2009;21:322-328.

5. Hasse B, Ledergerber B, Furrer H, et al. Morbidity and aging in HIV-infected persons: the Swiss HIV cohort study. Clin Infect Dis 2011;53:1130-1139.

6. Guaraldi G, Orlando G, Zona S, et al. Premature age-related comorbidities among HIVinfected persons compared with the general population. Clin Infect Dis 2011;53:11201126.

7. O'Brien KK, Bayoumi AM, Strike C, Young NL, Davis AM. Exploring disability from the perspective of adults living with HIV/AIDS: development of a conceptual framework. Health Qual Life Outcomes 2008;6:76. 
8. O'Brien KK, Davis AM, Strike C, Young NL, Bayoumi AM. Putting episodic disability into context: a qualitative study exploring factors that influence disability experienced by adults living with HIV/AIDS. J Int AIDS Soc 2009;12:5.

9. Lenderking WR, Testa MA, Katzenstein D, Hammer S. Measuring quality of life in early HIV disease: the modular approach. Qual Life Res 1997;6:515-530.

10. Shumaker SA, Ellis S, Naughton M. Assessing health-related quality of life in HIV disease: key measurement issues. Qual Life Res 1997;6:475-480.

11. Hays RD, Shapiro MF. An overview of generic health-related quality of life measures for HIV research. Qual Life Res 1992;1:91-97.

12. Clayson DJ, Wild DJ, Quarterman P, Duprat-Lomon I, Kubin M, Coons SJ. A comparative review of health-related quality-of-life measures for use in HIV/AIDS clinical trials. Pharmacoeconomics 2006;24:751-765.

13. Carabin H, Sonleitner NK, Keesee M, Shinault K. Quality of life measurement tools for people living with HIV/AIDS. Journal of HIV/AIDS \& Social Services 2008;7:71-82.

14. O'Brien KK, Bayoumi AM, Strike C, Young NL, King K, Davis AM. How do existing HIV-specific instruments measure up? Evaluating the ability of instruments to describe disability experienced by adults living with HIV. Health Qual Life Outcomes 2010;8:88.

15. Duracinsky M, Herrmann S, Berzins B, et al. The development of PROQOL-HIV: an international instrument to assess the health-related quality of life of persons living with HIV/AIDS. J Acquir Immune Defic Syndr 2012;59:498-505.

16. Duracinsky M, Lalanne C, Le Coeur S, et al. Psychometric validation of the PROQOLHIV questionnaire, a new health-related quality of life instrument-specific to HIV disease. J Acquir Immune Defic Syndr 2012;59:506-515. 
17. O’Brien KK, Alexander, R, King, K, et al. Developing a new HIV disability questionnaire: a community-integrated approach. Physiotherapy Canada 2010;62:34-35.

18. O'Brien KK, Bayoumi AM, Bereket T, et al. Sensibility assessment of the HIV Disability Questionnaire. Disabil Rehabil 2013;35:566-577.

19. Pett MA, Lackey NR, Sullivan JJ. Making Sense of Factor Analysis: The use of factor analysis for instrument development in health care research. California: Sage Publications, Inc; 2003.

20. O'Brien K. Factor analysis: an overview in the field of measurement. Physiother Canada 2007;59:142-155.

21. Watson R. Publishing the results of factor analysis: interpretation and presentation. J Adv Nurs 1998;28:1361-1363.

22. Kaiser HF. An index of factorial simplicity. Psychometrika 1974;39:32-36.

23. Floyd FJ, Widaman KF. Factor analysis in the development and refinement of clinical assessment instruments. Psychological Assessment 1995;7:286-299.

24. Horn JL, Engstrom, R. Cattell's scree test in relatoin to Bartlett's chi-square test and other observations on the number of factors problem. Multivariate Behavioral Res. $1979 ; 14(3): 283-300$.

25. Costello AB, Osborne JW. Best practices in exploratory factor analysis: Four recommendations for getting the most from your analysis. Practical Assessment, Research and Evaluation 2005;10:1-9.

26. Cattell RB. The scree test for the number of factors. Multivariate Behav Res 1966;1:245276.

27. Kline P. An Easy Guide to Factor Analysis. New York: Routledge; 1994.

28. SAS Institute Inc. SAS Computer Software 9.2. 2008. 
29. IBM Corp. SPSS Statistics for Windows, Version 19.0. Armonk, NY: IBM Corp; 2010.

30. Hatcher L. A step-by-step approach to using SAS for factor analysis and structural equation modeling. Cary, NC, USA: SAS Institute Inc.; 1994.

31. Carter SL, Rourke SB, Murji S, Shore D, Rourke BP. Cognitive complaints, depression, medical symptoms, and their association with neuropsychological functioning in HIV infection: a structural equation model analysis. Neuropsychology 2003;17:410-419.

32. Marx RG, Bombardier C, Hogg-Johnson S, Wright JG. Clinimetric and psychometric strategies for development of a health measurement scale. J Clin Epidemiol 1999;52:105111.

33. Feinstein AR. Benefits and obstacles for development of health status assessment measures in clinical settings. Med Care 1992;30:MS50-56.

34. Rourke SB, Bekele TM, Tucker R, et al. Housing affordability and sense of community belonging predict health-related quality of life among people with HIV in Ontario:

Results from the Positive Spaces, Healthy Places study. Can J Infect Dis Med Microbiology 2011;22.

35. Streiner DL, Norman GR. Health Measurement Scales: A practical guide to their development and use. Fourth edn. Oxford, New York: Oxford University Press; 2008.

36. Bollen KA, Barb KH. Pearson's $r$ and coarsely categorized measures. Am Sociol Rev $1981 ; 46: 232-239$.

37. ten Holt JC, van Duijn MAJ, Boomsma A. Scale construction and evaluation in practice: A review of factor analysis versus item response theory applications. Psychological Test and Assessment Modeling 2010;52:272-297.

38. Mayo NE, Wood-Dauphinee S, Ahmed S, et al. Disablement following stroke. Disabil Rehabil 1999;21:258-268. 
39. Wilson IB, Cleary PD. Linking clinical variables with health-related quality of life. A conceptual model of patient outcomes. JAMA. 1995;273:59-65.

40. de Vet HC, Ader HJ, Terwee CB, Pouwer F. Are factor analytical techniques used appropriately in the validation of health status questionnaires? A systematic review on the quality of factor analysis of the SF-36. Qual Life Res 2005;14:1203-1218; dicussion 1219-1221, 1223-1204.

URL: http:/mc.manuscriptcentral.com/dandr Email: davemuller@suffolk.ac.uk 